\title{
Route Optimization in NEMO Environment with Limited Prefix Delegation Mechanism^
}

\author{
Jungwook Song, Sunyoung Han ${ }^{\star \star}$, and Kiyong Park \\ College of Information and Communication \\ Department of Computer Science and Engineering \\ Konkuk University \\ 1 Hwayang, Gwangjin, Seoul 143-701, Korea \\ \{swoogi, syhan, kypark\}@cclab.konkuk.ac.kr
}

\begin{abstract}
The $4 \mathrm{G}$ network will must be ALL-IP network and IPv6 is destiny. Already IPv6 has extended as Mobile IPv6 for host mobility and Mobile IPv6 is extending for whole network mobility. There are many possible mobile networks, and they would be nested as they change locations. Most serious problem in a nested mobile network is the complexity of routing path of packets, and the complexity grows as the level of nesting increases. In this paper, we propose 'limited prefix delegation mechanism', which delivers packets through the optimized path even though mobile networks are nested. We show the effectiveness of our mechanism by solved problems.
\end{abstract}

\section{Introduction}

As information and communication technologies are rapidly progressed, there are increasing sorts of informative devices that can access wireless network while they move their locations. There are many researches on wireless and mobile network. When IPv6 is widely deployed, almost electronic devices will be connected to the Internet. These two key cores the 'Mobile Access Network' and 'IPv6', are necessary for the 'Ubiquitous Computing'.

If IPv6 prevails and mobile access network is established, it would be a common case that tens or hundreds of mobile nodes change their locations at the same time. Because existing Mobile IPv6 has been designed to support host mobility only [1, they do not smoothly support the current movement of many hosts, i.e. movement of network that consisted with two or more hosts or PANs(Personal Area Networks) or network in moving vehicles. To support movement of whole network, IETF nemo WG is making study an extension of Mobile IPv6 and already has the protocol standard that called the 'NEMO Basic Support Protocol' 2].

\footnotetext{
* This research was supported by the MIC(Ministry of Information and Communication), Korea, under the ITRC(Information Technology Research Center) support program supervised by the IITA(Institute of Information Technology Assessment).

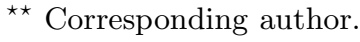


In general situation, however, any networks and hosts can change their location simultaneously, and they can be nested. Direct application of [2] to the nested mobile network causes complicated routing path from MNN(Mobile Network Node) to the $\mathrm{CN}$ (Correspondent Node). Therefore, it is important to optimize the routing path of packets in nested mobile network. The route optimization problem is a part of main issues that working progress 3, 4. The problems of nested mobile network are described in [3].

In this paper, we try to solve the route optimization problem in nested mobile network using the 'limited prefix delegation mechanism'. Instead of by opening bi-directional tunnel between the MR(Mobile Router) and its HA(Home Agent), the packet routing path can be optimized by opening tunnel directly from the $\mathrm{MR}$ to the $\mathrm{CN}$. And the $\mathrm{CN}$ sends packet with the routing header. Our mechanism opens one or two tunnels, and we can optimize the routing path in both directions.

\section{Limited Prefix Delegation Mechanism}

The 'limited prefix delegation mechanism' we propose in this paper differs from [2]. Our mechanism sends all packets directly through tunnel between the MR and the CN. The packet from MNN to the CN is tunneled on MR, and the CN sends the packet to the MNN with the routing header. To achieve this, we add new RA(Router Advertisement) option on MR. And we also modified Binding Update process and tunneling process on MR.

\subsection{Addition of New Router Advertisement Option}

To all MRs in nested mobile network open just one tunnel to the CN directly, delegating access router's prefix is required. The MR that attached access router directly, it is simple that delegating prefix. But, the nested MRs cannot recognize access router's prefix. So, Upper link MR must notify access router's prefix to nested MRs. To achieve this, we add new RA option called 'delegated prefix option' as shown in Fig. 1.

If the MR does not receive this option, MR delegating prefix from uplink router's(access router's) original RA message, and install new care-of address on

\begin{tabular}{|l|c|c|c|c|c|c|}
\hline Length & Prefix Length & L & A & R & D & Reserved1 \\
\hline \multicolumn{4}{|c|}{ Valid Lifetime } \\
\hline \multicolumn{4}{|c|}{ Preferred Lifetime } \\
\hline Reserved2 \\
Delegated Prefix \\
\hline
\end{tabular}

Fig. 1. Delegated Prefix Option 
its egress interface. And the MR adds delegated prefix option to its RA message. The new RA message consists of prefix option that has prefix of home link, and delegated prefix option that has delegated prefix from access router.

If the MR receives delegated prefix option, MR delegating prefix from this delegated prefix option, and then same as before case. So, all nested MR can have care-of address that is subnet address of visited access router on top of nested mobile network. And, all other MNNs silently ignore delegated prefix option from RA message, thus there are no changes on MNNs.

\subsection{Modification of Tunneling on Mobile Router}

In our mechanism, the MR sends packet through direct tunnel to the CN instead of sending through the tunnel to its HA. If outgoing packets have source address with mobile network prefix(from home link prefix), the MR should open direct tunnel to the CN. In this scheme, the MRs do not care about the types of MNN, whether it is plain host or not: it applies the same scheme on all packets passing by that have source address with mobile network prefix.

\subsection{Modification of Binding Update Process}

When a MR detects its movement, it performs Binding Update process. In the same way, the MR sends Binding Update to the $\mathrm{CN}$ when a MNN sends packets to a $\mathrm{CN}$ or a $\mathrm{CN}$ sends packets to a MNN. The $\mathrm{CN}$ that received Binding Update can send packets to the MR directly. The MR maintains the ' $\mathrm{CN}$ table' to keep the information if Binding Update was sent to the $\mathrm{CN}$ or not. The MR must initialize this table when it moves to another location.

\subsection{Processing Routing Header on Mobile Router}

If the CN was enabled Mobile IPv6 and received Binding Update from the MR, the CN sends packet to the MR's care-of address with the routing header type 2 , that has the MNN's original address. When the MR receives this packet, it examines that packet has the routing header, and if exists, the MR forwards packet to the MNN that is original destination.

\section{Solved Problems}

There are many cases of routing problem in nested mobile network environment. With our mechanism proposed in this paper, we can solve many problems. The mobile network configurations and its problems are described in [3], so you must refer 'Appendix B' of that document parallel with this section.

When apply our mechanism to the Case A, MR3 opens tunnel to CN directly, so packets from LFN to $\mathrm{CN}$ are passing by optimized path. And there is no mobility functions on CN, so CN sends packets to MR3_HA, and MR3_HA can open tunnel to MR3 directly. We can achieve near-optimized path. When apply our mechanism to the Case B, MR3 opens tunnel to CN directly, so packets 
from LFN to $\mathrm{CN}$ are passing by optimized path. And $\mathrm{CN}$ has mobility function; CN sends packets to MR3 directly with the routing header. Thus, we can achieve optimized path in both directions. The Case C, after Mobile IPv6 route optimization, the rest are same processes as Case A.

The Case D is similar to Case A. When apply our mechanism in this- case, MR3 try to opening tunnel to CN directly, and MR5_HA receives and forwards it via bi-directional tunnel between MR5_HA to MR5. When MR5 receives packet from MR3, MR5 send Binding Update to MR3. So, MR3 sends packet directly to MR5 with the routing header. The same process occurs on reverse direction. Thus, we can achieve optimized path in both directions with only two tunnels. The Case E is similar to Case B and The Case F is similar to Case C. After Mobile IPv6 route optimization, the rest are same processes as Case D.

The Case G is similar to Case A. Same process occurs as Case D. And the Case H, same process occurs as Case E. The Case I, as for Case C and Case F, Mobile IPv6 Route Optimization cat not performed. But, MR3 and MR5 perform Route Optimization like Case D.

The Case J, no special function is necessary for optimization of their communication. The Case $\mathrm{K}$ is similar to Case $\mathrm{H}$. Two nodes may initiate Mobile IPv6 route optimization. Same as Case J, no special function is necessary. The Case L, Mobile IPv6 Route Optimization cannot be preformed. With our mechanism, similar to Case D, MR3 and MR5 send Binding Update to each other. Thus, we can achieve optimized path in both directions.

\section{Concluding Remarks}

In this paper, we proposed the 'limited prefix delegation mechanism' which optimizes the routing path in nested mobile network environment. From previous section, almost problems in [3] could be solved by our proposed mechanism.

The 'limited prefix delegation mechanism' opens just one or two tunnels, and it establishes direct path bypassing MRs' HAs. To achieve the optimized path, we add new 'delegated prefix option' on the MR's RA message, modify tunneling target on the MR, modify Binding Update process on the MR. We just add or modify small functions on the MR only. So, we can get much better results with little changes.

\section{References}

1. D. Johnson, C. Perkins, J. Arkko: Mobility Support in IPv6: RFC3775, IETF, 2004

2. V. Devarapalli, R. Wakikawa, A. Retrescu, P. Thubert: Network Mobility (NEMO) Basic Support Prototol: RFC3963, IETF, 2005

3. C. Ng, P. Thubert, M. Watari, F. Zhao: Network Mobility Route Optimization Problem Statement: IETF nemo WG Draft, 2005

4. C. Ng, F. Zhao, M. Watari, P. Thubert: Network Mobility Route Optimization Solution Space Analysis: IETF nemo WG Draft, 2005 